\title{
Evaluating Natural Alternatives to Synthetic Acrylic Polymers: Rheological and Texture Analyses of Polymeric Water Dispersions
}

\author{
Giovanni Tafuro,* Alessia Costantini, Gianni Baratto, Stefano Francescato, and Alessandra Semenzato
}

Cite This: https://dx.doi.org/10.1021/acsomega.0c01306

Read Online

ABSTRACT: The natural cosmetics market is growing, in line with the interest of public opinion on environmental safety. The availability of polysaccharides for cosmetic use is very wide; each raw material has its own sensorial specificities and hardly matches the performance of synthetic polymers. We developed an instrumental protocol based on rheology and texture analysis to evaluate alternatives to acrylic polymers. The study has been carried out on a set of water dispersions prepared with different synthetic, semisynthetic, and natural polymers at different concentrations. Using statistical principal component analysis, three different clusters have been identified: group A includes polymers with a stringy viscoelastic behavior, group B includes polymers with low firmness and a weak-gel rheological pattern, and

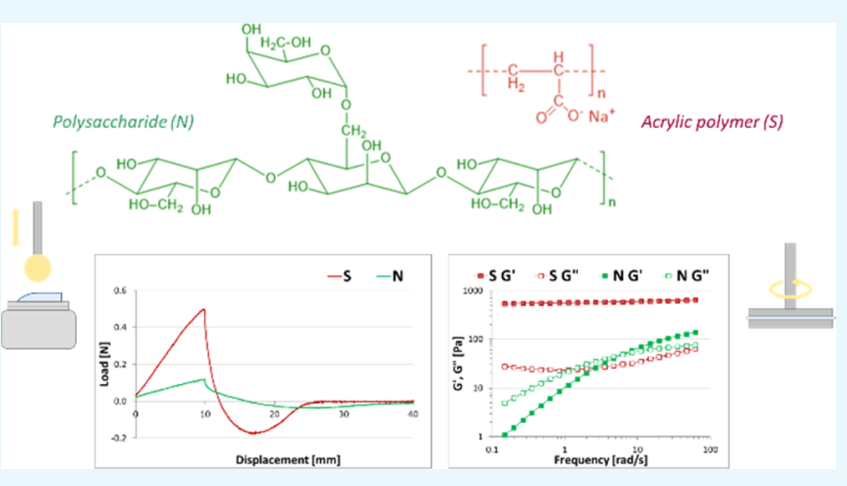
group $\mathrm{C}$ includes polymers which formed soft and elastic gels. This work showed that this instrumental approach is a powerful tool to comprehensively characterize new rheological modifiers and to forecast their contribution to the formulation based on their applicative features. Moreover, rheology and texture analysis turned out to be complementary tools useful to compare polymeric raw materials and to identify appropriate alternatives to synthetic ones in order to formulate green cosmetic products.

\section{INTRODUCTION}

Polymers are multifunctional ingredients widely used in cosmetic formulas for different purposes, such as thickening, emulsifying, stabilizing, and enhancing the product texture properties. The most common polymers used for these aims are the synthetic ones belonging to the family of polyacrylates, which are versatile and high-performing materials. ${ }^{1}$ Nowadays, the interest on natural polymers is increasing, together with a major interest for environmental issues and sustainability that is becoming an imperative for the cosmetic companies which must be aware of the environmental impact of the whole life

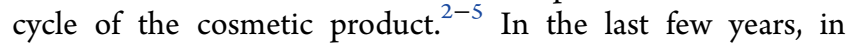
Europe, there has been a heated debate on the topic of microplastics and the damage of the aquatic ecosystem: these solid and spherical plastic particles less than $5 \mathrm{~mm}$ in size are banned from cosmetic products. ${ }^{6}$ Besides that, there is a growing concern on the overall impact of liquid plastics, such as polyacrylic polymers, which can be dispersed or emulsified in cosmetic formulas with functional purposes. ${ }^{7}$ These polymeric materials are also mainly responsible for the texture tactile properties of skin care products. ${ }^{8,9}$ It is well-known that consumers immediately evaluate the sensory characteristics of the product when applied on the skin, and this aspect is the main driver of the purchase process. ${ }^{10}$ For this reason, it is important for cosmetic industries to find viable natural alternatives, without losing the sensorial characteristics and the functional-stability aspects impaired by synthetic polymers.
Xanthan gums, celluloses, starches, and gums are widely used ingredients not only in the natural cosmetic market but also in food, pharmaceutical, and adhesives industries. Polysaccharides are complex carbohydrates which, according to their chemical structure, can influence the mechanical properties of the system. ${ }^{11}$ In fact, they are characterized by the presence of many hydroxyl groups which, once dispersed in water, can strongly interact with water molecules and increase the viscosity of the system. ${ }^{12}$ From a technical point of view, replacing high-performing synthetic polymers in formulas with natural alternatives can be quite challenging, and considering the wide variety of plant-based polysaccharides available for cosmetic use, manufacturers are continuously more interested in instrumental methodologies to be used as time-saving and reproducible prescreening tools to evaluate the applicative specificities of new natural ingredients and assess the product design.

The importance of rheology for the characterization of semisolid materials has been widely proven. In particular, the

Received: March 24, 2020

Accepted: June 3, 2020 


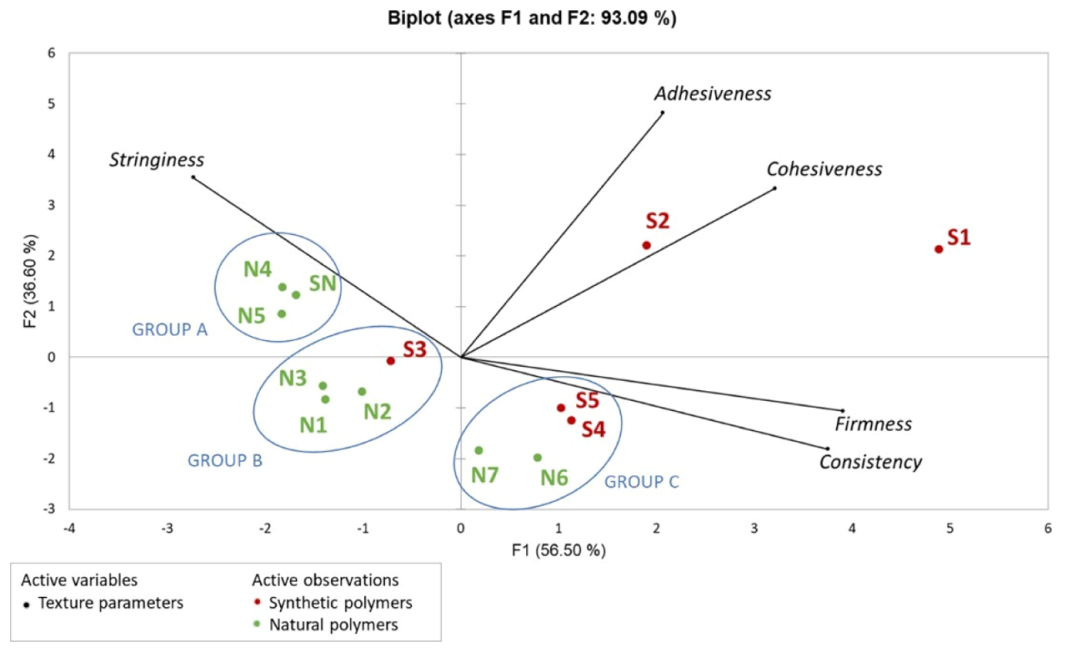

Figure 1. PCA loading biplot of the textural parameters (variables) measured with the texture analyses of the samples (observations), using the first principal component $\left(F_{1}\right)$ and the second principal component $\left(F_{2}\right)$.

results obtained from the analyses, conducted both in continuous and oscillatory flow conditions, are used in the research and development of cosmetics, to investigate the spreading properties of products, their internal structure, and physical stability. ${ }^{13,14}$ The texture analysis is frequently used in the food sector for determining the textural properties of foods. ${ }^{15-17}$ In recent times, this instrumental test has been also introduced in the cosmetic industry to quantify multiple textural properties, which are related to the sensory characteristics of products, such as gels and emulsions. ${ }^{18}$ Recent studies have described the usefulness of rheology and texture measurements used in combination to compare different cosmetic products and to obtain objective information on their physical-mechanical attributes. ${ }^{19,20}$ This work is intended to investigate the rheological and textural properties of water dispersions prepared using various acrylic and natural-derived polymers at different concentrations. The textural properties known as firmness, consistency, adhesiveness, cohesiveness, and stringiness have been evaluated through an immersion/ deimmersion test by means of a texture analyzer. The rheological curves have been obtained with measurements conducted both in continuous and oscillatory flow conditions. To verify the existence of any correlations between these two instrumental techniques, the obtained data have been statistically analyzed.

\section{RESULTS AND DISCUSSIONS}

The dataset made of the polymer dispersions as observations and the texture attributes as variables has been statistically processed with principal component analysis (PCA), which allowed examining the differences between the mechanical characteristics of the samples prepared in this work and pointing out the relations that occur between the parameters investigated (Figure 1). In order to better visualize the properties conferred to the system by the raw materials and to get as close as possible to the conditions of actual use in the formulation, only the representative concentration of each polymer, among the concentration range indicated in the technical data sheets, was reported in the PCA graph. The first axis $F_{1}$ represented the stiffness and the solidity of the analyzed systems because it was positively related to the parameters of firmness, consistency, cohesiveness, and adhesiveness. The second axis $F_{2}$, instead, which was positively related to stringiness and negatively related to all the other variables, could represent product picking and film-forming properties. In detail, adhesiveness, cohesiveness, and stringiness were positively related to $F_{2}$, whereas firmness and consistency were negatively related to it. The positioning of the polymeric raw materials inside the PCA plot was a consequence of the physical-mechanical properties they gave to the aqueous systems in which they were dispersed. From a general point of view, there was a clear distinction between the synthetic polymers and the natural-derived ones. A majority of the natural raw materials were positioned on the left side of the graph because they had lower values of firmness, consistency, cohesiveness, and adhesiveness and were more likely to form fluid systems. On the contrary, the major part of the synthetic polymers was distributed in the right area of the graph because they were characterized by higher textural parameters than the polysaccharides except for stringiness, indicating the ability to form stiffer gelled systems.

The synthetic polymers S1 (carbomer) and S2 (sodium polyacrylate) are positively loaded on both $F_{1}$ and $F_{2}$. They are high molecular weight polymers derived from the crosslinking of the polyacrylic acid. Acrylic polymers when dispersed in water and neutralized with a common base such as sodium hydroxide $(\mathrm{NaOH})$, converting the acidic polymer into a salt, form a three-dimensional network: the polymer chains begin to swell and partially uncoil because of electrostatic repulsion among the negative charges, conferring high viscosity, consistency, and shear thinning characteristics to aqueous systems. These polymers differ for the position on $F_{1}$, probably due to the different crosslinking degree and the conformation of the microgel structure. S1, in particular, leads to the formation of stiffer hydrogels, resistant to external deforming forces. It registered high levels of firmness, consistency, cohesiveness, and adhesiveness, and for this reason, it is located on the very right side of the graph. The dispersion made of S2, which is a preneutralized form of the acrylic polymer, showed high values of cohesiveness and adhesiveness but lower firmness. The textural and the rheological profiles of the samples prepared by dispersing these acrylic polymers in water were deeply investigated in our previous work. ${ }^{21}$

The other polymers analyzed in this work can be split into three distinct clusters. SN (hydroxypropyl guar gum), N4 ( 
Caesalpinia spinosa gum), and N5 (Ceratonia siliqua gum) belong to the same cluster (group A), negatively loaded on $F_{1}$ and positively loaded on $F_{2}$. They are characterized by the highest values of stringiness and the lowest values of consistency and firmness. The latter is a parameter described as the force needed to reach a certain degree of deformation, and it is directly connected to the product spreadability. The lower the firmness, the easier the application because less force is needed to make the product flow on the skin. ${ }^{9}$ The dispersions formulated with these natural-derived raw materials are fluid systems with high spreadability and good pickup properties.

The synthetic polymer S3 (polyacrylate cross-polymer-11) and the natural polymers N1 (xanthan gum), N2 (succinoglycan), and N3 (sclerotium gum) form a second cluster (group B) because they are negatively loaded on $F_{1}$ and on $F_{2}$ too. These materials dispersed in the solution formed weak interactions with water, determining the formation of systems with medium-low textural parameters. Even if they belong to the same cluster, they differ from each other for their values on $F_{1}$. Among the materials belonging to this group, in fact, the synthetic polymer S3 showed the highest adhesive and cohesive properties, and this is a consequence of the stronger molecular interactions between the polymeric chains which formed the three-dimensional gelled network.

A third cluster (group C) is made of the hydrophobically modified synthetic polymers S4 (ammonium acryloyldimethyltaurate/beheneth-25 methacrylate cross-polymer) and S5 (acrylates/C10-30 alkyl acrylate cross-polymer) and the polysaccharides N6 (gellan gum) and N7 (carrageenan). These samples, positively loaded on $F_{1}$ and negatively loaded on $F_{2}$, were characterized by the presence of stronger intermolecular interactions, and for this reason, they showed high firmness and consistency but the lowest values of adhesiveness and stringiness. These parameters are typical of no-stringy bouncy systems with low pickup properties.

Group A. The polymers included in this group (SN, N4, and N5) when dispersed in water formed fluid systems, whose predominant textural characteristic is stringiness because a strand was formed during the withdrawal of the probe from the analyzed sample.

Table S1 presents the numerical values of the five textural parameters measured with an immersion/deimmersion test conducted with a texture analyzer on the samples prepared with different concentrations of the raw materials belonging to group A. As expected, increasing the polymer amount, an increase of the values of firmness, consistency, cohesiveness, and adhesiveness was registered. On increasing the polymer concentration, the force needed to deform the system was higher because more polymer-solvent interactions were formed, and the three-dimensional network became more and more structured. The polymer concentration also had an impact at the end of the texture curve: the stringiness increased at higher concentrations. These results obtained for the stringiness differ from the results obtained in our previous work for the hydrophilic gels prepared with synthetic acrylic polymers, whose stringiness decreased with increasing polymer concentrations. $^{21}$ The high values of stringiness shown by SN and the materials belonging to the same cluster are related to the sensorial expression of "long flow" used in formulation practice, opposite to that of "short flow" which indicates a cosmetic system whose overall rigidity is due to the presence of a highly crosslinked rheological modifier, which determines a reduction in stringiness. ${ }^{22}$

A comparison between the curves obtained from the texture analyses of the dispersions prepared with SN (hydroxypropyl guar gum), N4 (C. spinosa gum), and N5 (C. siliqua gum) at the same concentration of $1.5 \% \mathrm{w} / \mathrm{w}$ is reported in Figure 2.

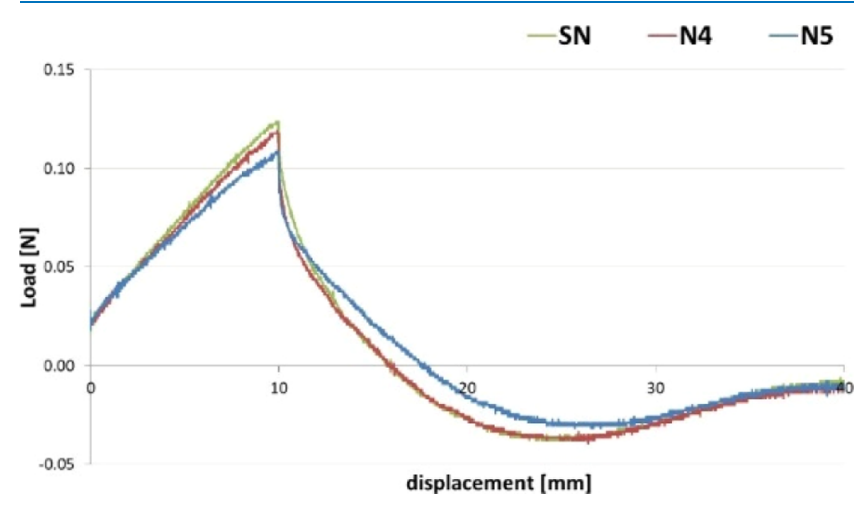

Figure 2. Textural profile of the samples prepared with $1.5 \% \mathrm{w} / \mathrm{w}$ of polymers $\mathrm{SN}, \mathrm{N} 4$, and N5. Data plotted as load vs cumulative displacement.

The samples showed a similar textural behavior. They had almost the same values of stringiness $(>20 \mathrm{~mm})$ because the three curves have the same endpoint on the graph. However, the sample prepared with N5 showed lower values of firmness, adhesiveness, and cohesiveness than the samples prepared with $\mathrm{N} 4$ and SN. We can assume that N5 in solution blindly interacts with water and is more likely to form liquid systems and fluid textures. The textural curves of $\mathrm{N} 4$ and $\mathrm{SN}$ are overlapped in the negative part, showing very similar values of adhesiveness and cohesiveness, but $\mathrm{SN}$ slightly reached higher values of firmness $(0.13 \mathrm{~N})$.

The controlled shear rate (CSR) test allowed studying the viscosity trend with an increasing shear rate. The viscosity values of the dispersions made with the polysaccharides of group A gradually decreased in function of the shear application, and this behavior is called shear-thinning. Figure 3 shows the flow curves of the samples prepared with an increasing concentration of $\mathrm{SN}$. The viscosity remained almost

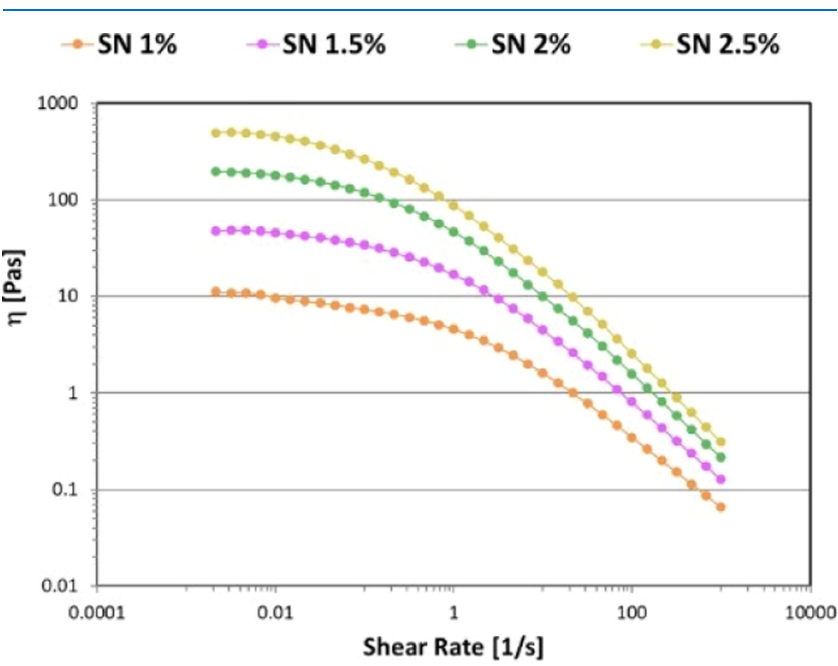

Figure 3. Viscosity curves in function of the shear rate applied for the samples prepared at increasing concentrations of $\mathrm{SN}$. 
a

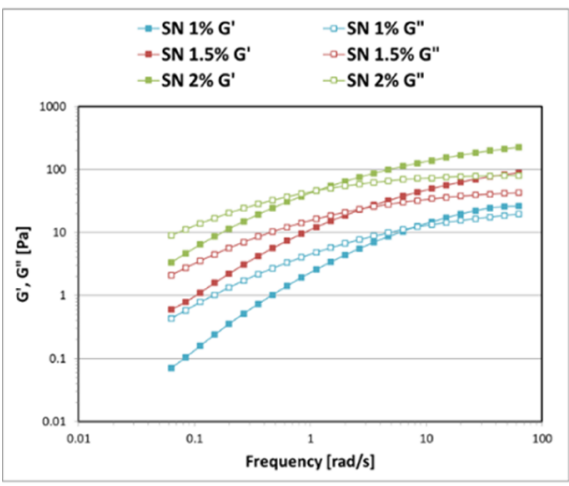

b

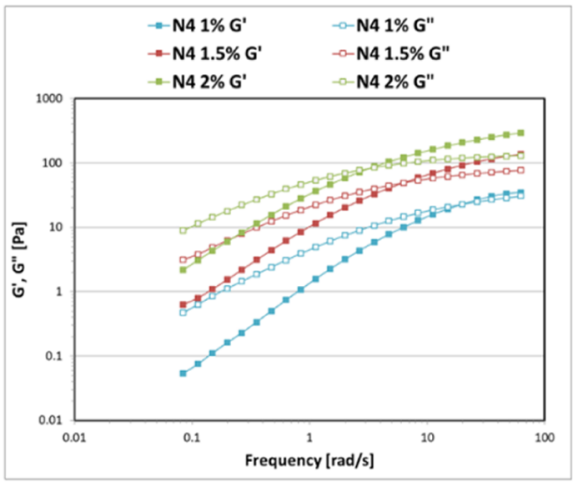

C

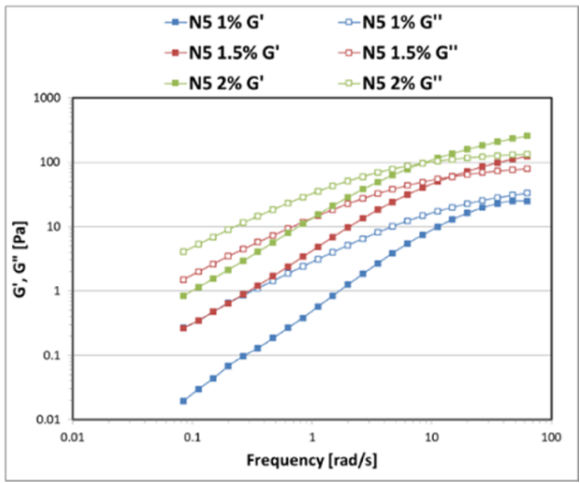

Figure 4. Trend of elastic $G^{\prime}$ and viscous $G^{\prime \prime}$ moduli in function of the frequency for the samples prepared with increasing concentrations of polymers SN (a), N4 (b), and N5 (c).

unchanged at lower shear rates, forming a Newtonian plateau region. As the shear rate increased, the viscosity was reduced because the polymer chains tended to align toward the flow direction. The viscosity of SN dispersions increased with the polymer concentration as a result of the stronger interactions between the polysaccharidic chains and the solvent. Table S2 reports the viscosity at rest $\left(\eta_{0}\right)$ values, calculated with the Carreau-Yasuda model, of the samples prepared with the three polymers belonging to group A. One can note that N5 dispersions reached lower values of firmness than the other two materials, in accordance with the lower textural values.

The viscoelastic properties as a function of frequency, using a fixed oscillation amplitude within the linear viscoelastic range, have been investigated (Figure 4). SN solutions showed that both the storage $G^{\prime}$ and the loss $G^{\prime \prime}$ moduli were significantly dependent on the frequency with a cross-over point, which is characteristic of the viscoelastic materials (Figure 4a). The viscous $G^{\prime \prime}$ modulus was predominant at lower frequencies and the storage $G^{\prime}$ modulus became higher than the viscous modulus when high frequencies were applied. By increasing the polymer concentration, the elastic character of the systems increased, and the frequency value correspondent to the cross-over point decreased (cross-over point frequency values: SN $1 \% 7.67 \mathrm{rad} / \mathrm{s}, \mathrm{SN} 1.5 \% 2.89 \mathrm{rad} / \mathrm{s}$, and SN 2\% $1.13 \mathrm{rad} / \mathrm{s}$ ). The water dispersions made of N4 showed a viscoelastic behavior similar to that of $\mathrm{SN}$ dispersions, but the frequencies corresponding to the cross-over point were higher, thus indicating a more liquid-like behavior than $\mathrm{SN}$ (cross-over point frequency values: N4 1\% $20.02 \mathrm{rad} / \mathrm{s}, \mathrm{N} 41.5 \% 6.28 \mathrm{rad} /$ $\mathrm{s}$, and N4 2\% $3.12 \mathrm{rad} / \mathrm{s}$ ) (Figure 4b). N5 solutions showed a transition from viscous liquid to a viscoelastic material as a function of the polymer concentration (Figure 4c). Up to $1 \%$, the viscous $G^{\prime \prime}$ modulus was greater than the elastic $G^{\prime}$ modulus in all of the frequency range investigated. By increasing the polymer concentration at $1.5 \%, G^{\prime}$ became higher than the viscous modulus when high frequencies were applied, showing a cross-over point (cross-over point frequency values: N5 1.5\% $14.92 \mathrm{rad} / \mathrm{s}$ and N5 2\% $8.38 \mathrm{rad} / \mathrm{s}$ ).

In Figure 5 the viscoelastic properties measured varying the frequency values of the water dispersions prepared at $1.5 \% \mathrm{w} /$ w of SN, N4 and N5, are compared. The damping factor $\tan \delta$ is calculated from the ratio between the loss modulus $G^{\prime \prime}$ and the storage modulus $G^{\prime}$, and it represents a way to describe the weight of the elastic components. N5 dispersion had the highest values of $\tan \delta$, having a predominant viscous behavior,

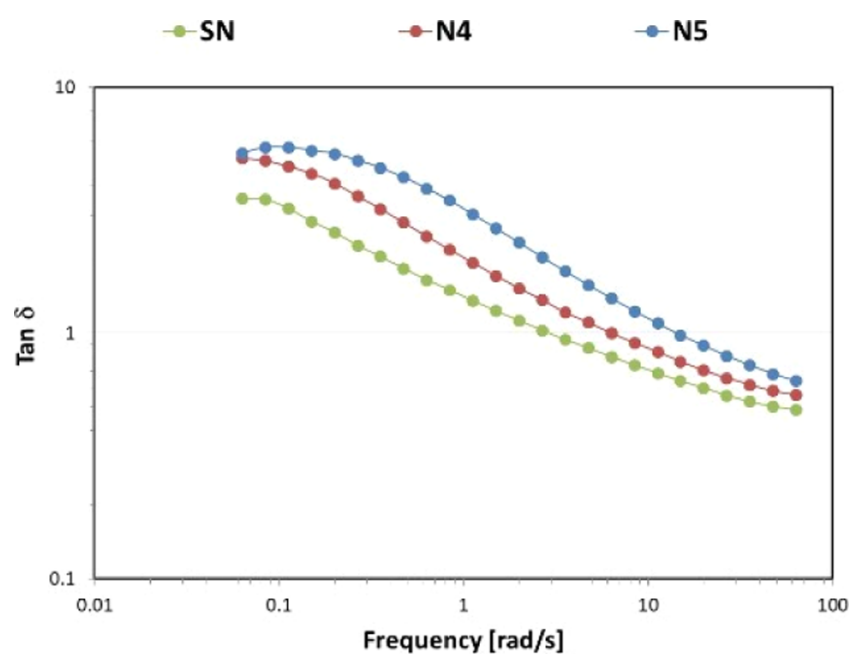

Figure 5. Damping factor $(\tan \delta)$ in function of the frequency for the samples prepared with polymers $\mathrm{SN}, \mathrm{N} 4$, and N5 $(1.5 \% \mathrm{w} / \mathrm{w})$.

and this indicated a higher dissipative capacity than the other materials. SN was characterized by the lowest values of $\tan \delta$ and a more elastic behavior.

These rheological modifiers are galactomannans consisting of a mannose backbone with side groups of galactoses branched on the six positions. These three glucomannans differ from their degree of substitution, that is, the number of side units per unit length of the main chain. The galactopyranose units prevent the formation of hydrogen bonds and inhibit the aggregation of the polysaccharidic main chains. N5 (C. siliqua gum, also known as the carob gum) is unevenly substituted because it has a ratio of $1: 4$ between mannose and galactose units. N4 (C. spinosa gum, also known as the tara gum) has a ratio of $1: 3$, while that of $\mathrm{SN}$ (hydroxypropyl guar gum) is of $1: 2 .^{23}$ For these reasons, SN can be easily dissolved in cold water, while N4 and N5 are less soluble and needed higher temperature to break the hydrogen bonds. The differences in the chemical structure also have an impact on the rheological and textural properties they impaired to aqueous dispersions. N5, in fact, showed lower viscosity and a more liquid-viscous rheological behavior than $\mathrm{S} 4$ and $\mathrm{SN}$, which have a higher substitution degree. Moreover SN, besides having more galactose side chains, is a chemically modified polysaccharide: the hydrophobic side chains which establish 
a

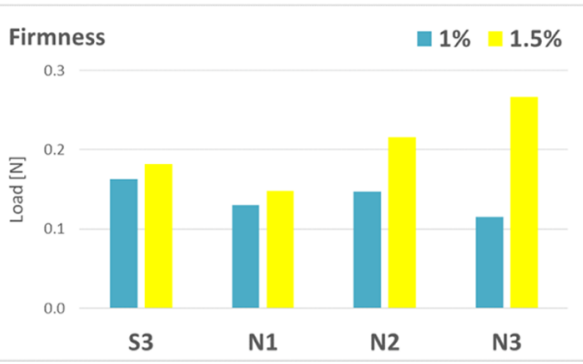

b

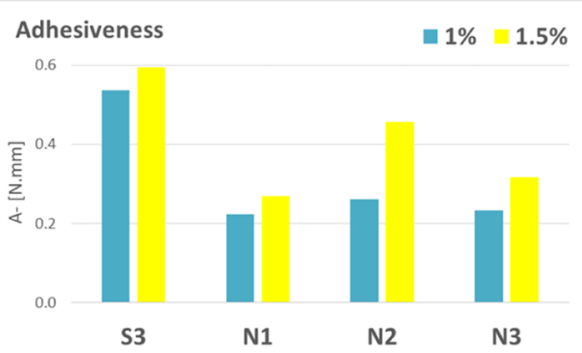

Figure 6. Textural parameters firmness (a) and adhesiveness (b) measured for the samples prepared with S3, N1, N2, and N3 at 1\% and 1.5\% w/w.

a

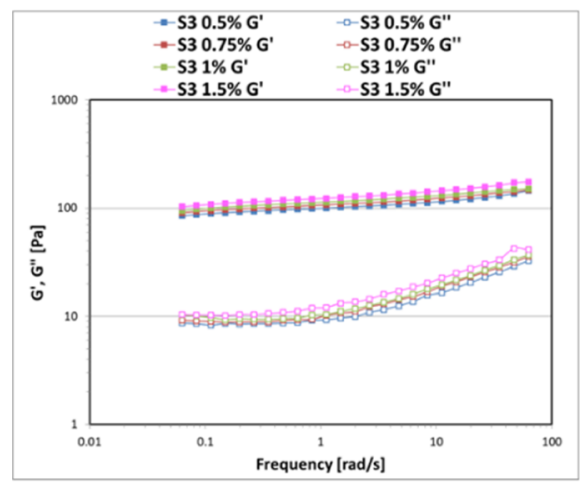

C

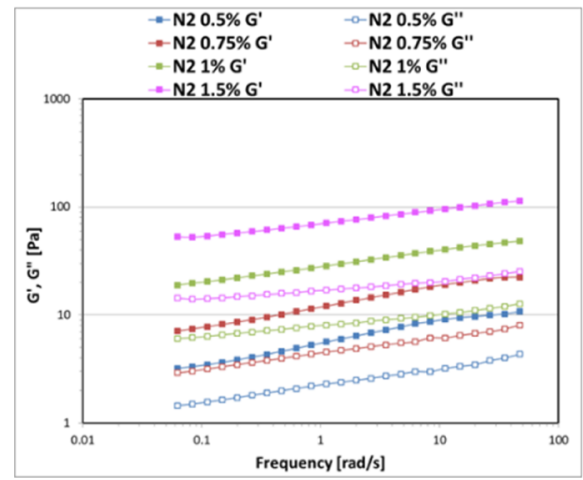

b

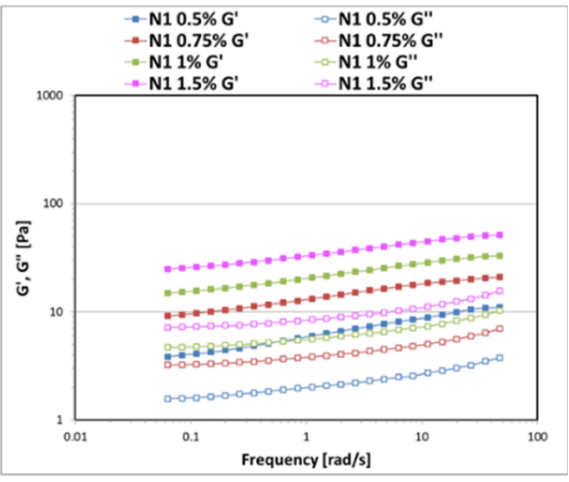

d

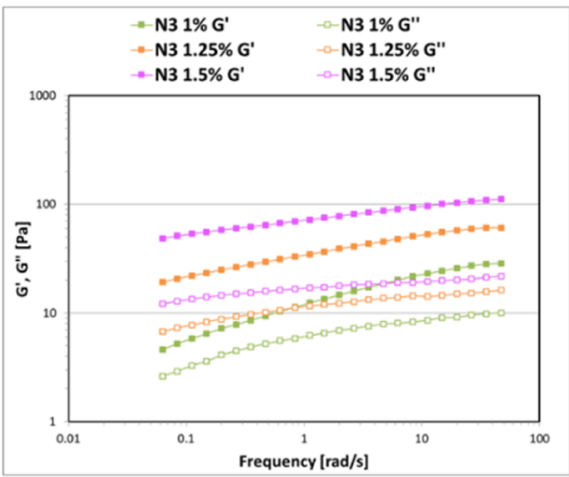

Figure 7. Trend of elastic $G^{\prime}$ and viscous $G^{\prime \prime}$ moduli in function of the frequency applied for samples prepared at increasing concentrations of $S 3$ (a), N1 (b), N2 (c), and N3 (d).

stronger intermolecular interactions lead to the formation of a system with higher viscosity.

Group B. The microbial-derived materials of this group, N1 (xanthan gum), N2 (succinoglycan), and N3 (sclerotium gum) and the synthetic polymer S3, when dispersed, determined the formation of weak interactions with water, forming aqueous systems with balanced textural parameters. In particular, they exhibited lower values of stringiness than the materials of group A. Table S3 reports the numerical values of the textural parameters measured through an immersion/deimmersion test performed on the samples prepared at increasing concentrations of the raw materials belonging to group $\mathrm{B}$. The instrument registered increasing values of firmness, consistency, and adhesiveness in function of the polymer concentration. On the other hand, stringiness decreased at increasing polymer concentrations, contrary to what happened for the raw materials of group A. The interactions between the polymers of group B and the solvent were stronger to that formed by the polymers of group A, determining a more structured, less deformable network. Figure 6 shows a comparison between the textural parameters of firmness (Figure 6a) and adhesiveness (Figure 6b) of the samples prepared at the same concentrations ( $1 \%$ and $1.5 \% \mathrm{w} / \mathrm{w})$ of the raw materials belonging to group $\mathrm{B}$.

The polymeric dispersions at $1 \% \mathrm{w} / \mathrm{w}$ showed values of firmness between 0.11 and $0.16 \mathrm{~N}$. The natural-derived raw materials $\mathrm{N} 2$ and $\mathrm{N} 3$, at higher concentrations $(1.5 \% \mathrm{w} / \mathrm{w})$, reached even greater values of firmness than the synthetic polymer S3. Among the natural polymers, N2 showed higher values of adhesiveness. However, the natural-derived materials did not reach the textural characteristics of the synthetic polymer S3 in terms of adhesiveness, even at higher concentrations. 
a

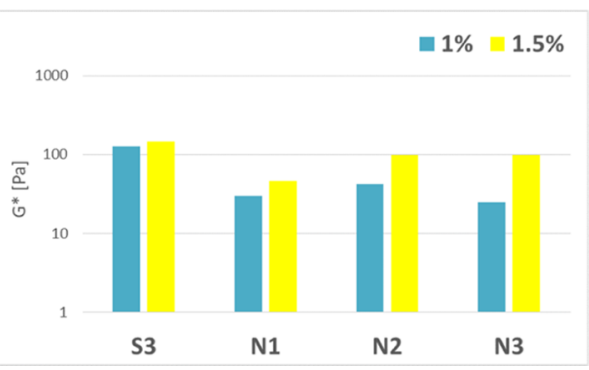

b

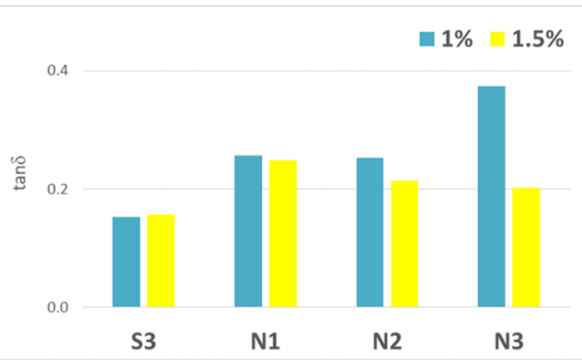

Figure 8. Comparison between the complex moduli $\mathrm{G}^{*}$ (a) and the damping factors $\tan \delta$ (b) calculated at a frequency of $1.7 \mathrm{~Hz}$ for the polymer $\mathrm{S} 3, \mathrm{~N} 1, \mathrm{~N} 2$, and $\mathrm{N} 3$ dispersed in water at a concentration of $1 \%$ and $1.5 \% \mathrm{w} / \mathrm{w}$.
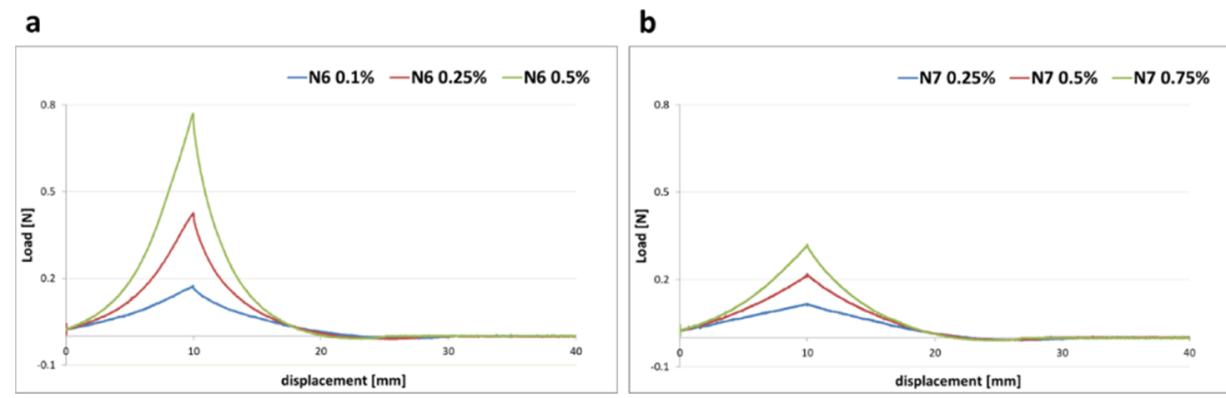

Figure 9. Texture profile curves obtained with an immersion/deimmersion test performed on the samples prepared with increasing concentrations of N6 (a) and N7 (b). Data plotted as load (N) vs cumulative displacement (mm).

The rheological analyses conducted in continuous flow conditions highlighted the shear-thinning behavior of the samples prepared with the polymers of group B (Figure S1), and the values of the zero-shear viscosity $\left(\eta_{0}\right)$ calculated with the Carreau-Yasuda model are reported in Table S4. The pseudo-plastic behavior of the polysaccharides N1, N2, and N3 was due to the rod-like conformation assumed in water: the highly ordered helices, stabilized by intramolecular H-bonds, were aligned toward the flux direction, determining a progressive decrease in viscosity when shear is applied.

The frequency sweep tests were conducted on the water dispersions choosing a strain value that falls within the linear viscoelastic region (LVR), in which both the elastic $G^{\prime}$ and viscous $G^{\prime \prime}$ moduli have a parallel trend independent of the strain. The mechanical spectra registered are shown in Figure 7.

$G^{\prime}$ was larger than $G^{\prime \prime}$ throughout the entire range of frequencies investigated. The moduli were parallel and dependent on the frequency applied and they settled between the first and the second decades. This behavior, typical of that of the weak gels, indicated that the elastic components always dominated over the viscous ones and that the system's structure was held by physical bonds between the macromolecules. By increasing the concentration of S3, there were only a few variations of the moduli values because the system was saturated at $0.5 \%$ of polymer concentration (Figure $7 \mathrm{a}$ ). For the natural polymers N1, N2, and N3, the higher their amounts, the higher the moduli (Figure $7 b-d$ ). In fact, increasing the polysaccharide concentration, the helicoidal structures became entangled, and a three-dimensional network was formed, in which water remained entrapped. The synthetic polymer S3 showed higher values of the complex modulus $G^{*}$, defined as the ratio between the total stress and the deformation to which the material is subjected at a given frequency. The natural polymers $\mathrm{N} 2$ and N3 used at a concentration of $1.5 \% \mathrm{w} / \mathrm{w}$ reached higher values of moduli than N1, close to that of the synthetic one S3 at $1 \% \mathrm{w} / \mathrm{w}\left(G^{*}\right.$ values at 1.7 Hz: S3 1\% 125.68 Pa, N1 1.5\% 46.35 Pa, N2 1.5\% 98.11 Pa, and N3 1.5\% 98.93 Pa) (Figure 8a), but they did not reach the $\tan \delta$ values of the synthetic polymer $\mathrm{S} 3$, which showed lower values, indicating a more elastic behavior, which was a consequence of the more complex crosslinked structure (Figure 8b).

The differences among these microbial-derived polysaccharides are due to the chemical structures and the different modality of interaction with water molecules. The xanthan gum has a cellulose-like backbone of glucose residues substituted with a trisaccharide side chain on alternating glucose units which consists of two mannose and a glucuronic acid residue. Noncarbohydrate substituents include an acetyl group on the inner mannose and a pyruvate group on the terminal mannose. The pyruvic acid content of xanthan can vary according to the producing bacterial strain and can affect the performance of the gum in water. Succinoglycan is an octasaccharide repeating unit consisting of seven glucose and one galactose with acetyl, pyruvyl, and succinyl substitutions. The long and flexible side chains of succinoglycan, which extrude from the double helices formed in solution, can establish more intermolecular interactions than the side chains of the xanthan gum determining the formation of stiffer gels. ${ }^{24}$ A sclerotium gum is a nonionic polysaccharide characterized by a linear chain of $(1,3)$ - $\beta$-D-glucose with a single $(1,6)-\beta$-Dglucose every third unit of the main chain. In water, it assumes a triple helix conformation held by intramolecular H-bonds, which determines a more rigid and elastic gel than the xanthan gum at high concentrations. ${ }^{25}$

Group C. For the raw materials of group C, which includes N6 (gellan gum), N7 (carrageenan), and S4 and S5 (crosslinked hydrophobically modified acrylic polymers), firmness and consistency were the predominant texture 
a

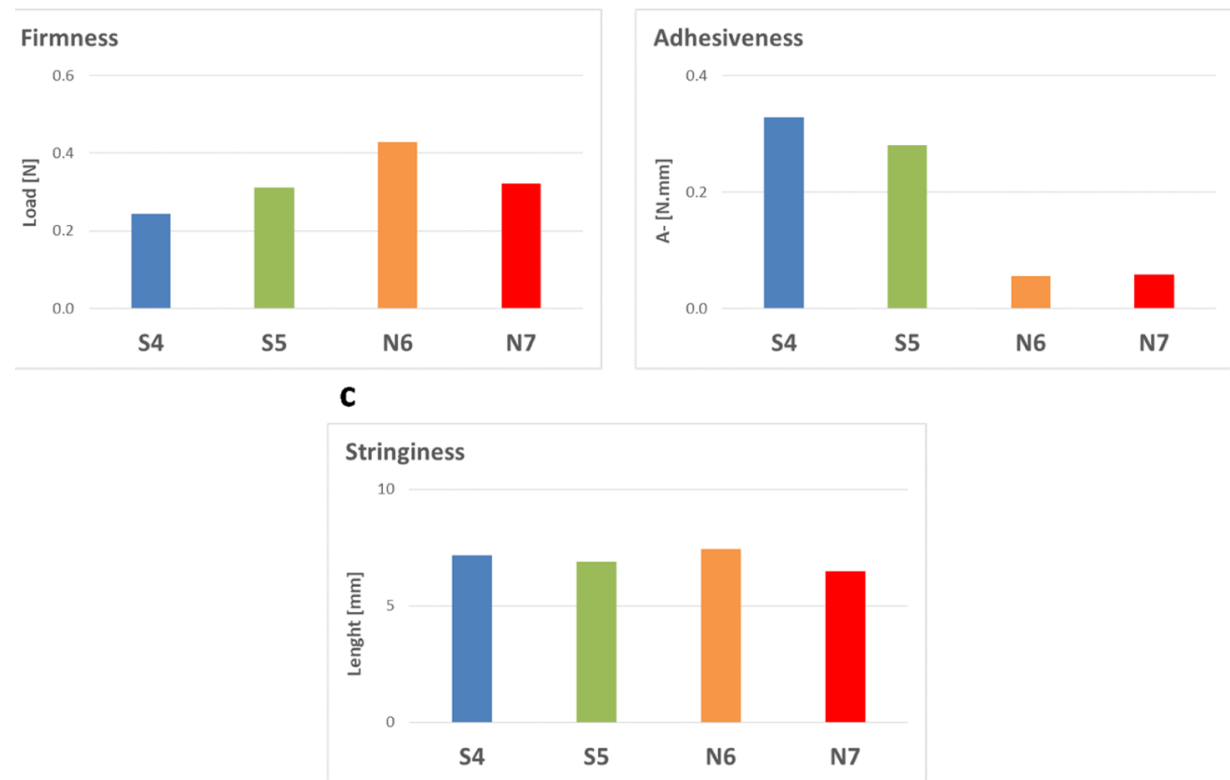

Figure 10. Textural parameters of firmness (a), adhesiveness (b), and stringiness (c) measured for the samples prepared with S4, S5, and N7 at $0.75 \% \mathrm{w} / \mathrm{w}$ and $\mathrm{N} 6$ at $0.25 \% \mathrm{w} / \mathrm{w}$.

a

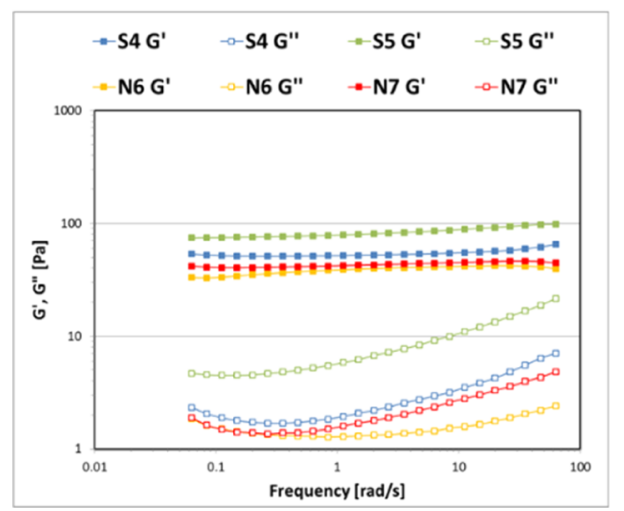

b

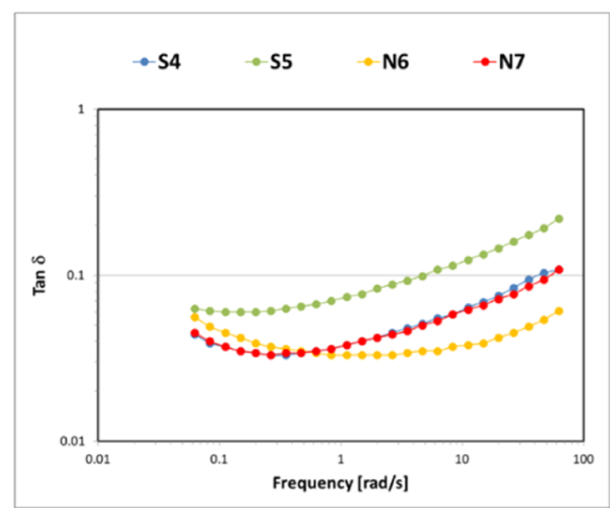

Figure 11. Trend of the elastic $G^{\prime}$ and $G^{\prime \prime}$ moduli (a) and of $\tan \delta(\mathrm{b})$ in function of the frequency applied for the samples prepared with S4, S5, N7 $(0.75 \% \mathrm{w} / \mathrm{w})$, and N6 $(0.25 \% \mathrm{w} / \mathrm{w})$.

parameters. Considering Table S5, the values of cohesiveness and adhesiveness were lower than firmness and consistency and remained almost constant at increasing concentrations. Stringiness decreased at increasing concentrations of polymers, as for the raw materials of group B. In Figure 9 the texture curves obtained with an immersion/deimmersion test performed on the samples prepared with increasing concentrations of the polysaccharides N6 and N7 are shown.

Both the polysaccharidic materials formed soft and elastic gels. Firmness and consistency increased at increasing polymer concentrations, maintaining low values of cohesiveness, adhesiveness, and stringiness, which is a typical profile of a gel with bouncy characteristics, poor film-forming properties, and low pickup. The positive curve can be divided into two areas: the immersion area (displacement from 0 to $10 \mathrm{~mm}$ ) and deimmersion area (displacement from 10 to $20 \mathrm{~mm}$ ). The ratio between the deimmersion area and the immersion area is called resilience. This is a textural parameter which indicates the ability of the sample to return to its original form after being deformed, compressed, or stretched. A complete resilient material has a resilience of 1 , whereas materials which modify their structure after applying an external force show values of resilience much lower than 1 , close to zero. The dispersions containing N6 and N7 showed high resilience (values between 0.8 and 0.9 ) because they were resistant to the deformation carried out by the probe and could almost completely recover their original shape. N6 showed higher values of firmness and consistency than N7 at the same concentrations. It is reasonable to think that the different structuring capabilities of these polymers are due to their different chemistry. N6 is a high acyl gellan gum consisting of a linear chain of glucose, rhamnose, and glucuronic acid (molar ratio 2:1:1). When dispersed in hot water in the presence of a monovalent salt, the polysaccharidic chains are uncoiled; after cooling, they assume a double helix conformation, stabilized by hydrogen bonds and by the presence of acyl groups, which determines the formation of a crosslinked network. ${ }^{26}$ On the other hand, $\mathrm{N} 7$ is an iota-carrageenan characterized by the presence of galactose dimers, with acidic sulfate groups. This polysaccharide forms double helices in water, stabilized by the presence of 
bivalent cations, which form cationic bridges between the negatively charged sulfate groups. ${ }^{27}$ As a result, the gels formed by the iota-carrageenan were less firm than those formed by the gellan gum, in which the physical interactions between the acyl ramifications lead to a more structured system even at lower concentrations. The systems formed by N6 $(0.25 \% \mathrm{w} /$ w) showed higher values of firmness not only than the gels formed by $\mathrm{N} 7$ at higher concentrations $(0.75 \% \mathrm{w} / \mathrm{w})$ but also than the gels formed by the synthetic acrylic polymers $\mathrm{S} 4$ and S5 $(0.75 \% \mathrm{w} / \mathrm{w})$ (Figure 10a). However, the synthetic polymers reached higher values of adhesiveness than N6 and N7 because it was more difficult to overcome the attractive forces between the sample and the probe (Figure 10b). The dispersions of the raw materials belonging to group $\mathrm{C}$ did not show significant differences in the values of stringiness (Figure 10c).

The flow curves obtained with a CSR test highlighted the pseudoplastic nature of the dispersions prepared with the polymers of group C (Figure S2), and the values of the zeroshear viscosity $\left(\eta_{0}\right)$ at increasing concentrations are reported in Table S6. Viscosity increased at increasing polymer concentrations, and the samples formulated with N6 showed the highest viscosity values. The frequency sweep rheological analyses conducted on the dispersions made with the raw materials of group $C$ revealed that the elastic $G^{\prime}$ modulus was higher than the viscous $G^{\prime \prime}$ modulus throughout the whole frequency range investigated. The moduli were located between the first and the second decade (Figure 11a). The synthetic polymer S5 (at $0.75 \% \mathrm{w} / \mathrm{w}$ ) reached higher values of moduli than the other rheological modifiers, but it showed a more prevalent viscous component, as indicated by the higher $\tan \delta$ values (Figure 11b). The natural-derived polymer N6 showed the lowest values of $\tan \delta$ especially when high frequencies were applied, indicating more elastic characteristics even at lower concentrations $(0.25 \% \mathrm{w} / \mathrm{w})$ in accordance with the higher values of firmness measured with the texture analysis. The polysaccharide N7 formed a gel with rheological properties similar to that formed by the synthetic polymer S4 at the same concentration $(0.75 \% \mathrm{w} / \mathrm{w})$ because the $\tan \delta$ curves were superimposed throughout the entire frequency range applied.

Correlations between the Textural and the Rheological Parameters. Pearson's coefficients have been calculated starting from the data obtained by measuring the textural and the rheological properties of the polymeric aqueous dispersions prepared and analyzed in this work. The textural parameters of firmness and consistency resulted to be positively correlated to the viscosity values obtained with the rheological tests conducted in continuous shear flow (Pearson's coefficient calculated for $\eta_{0}$ was 0.9 and for the $\eta$ at $0.1 \mathrm{~s}^{-1}$ was 0.8 ). The textural parameters of adhesiveness and cohesiveness were significantly correlated to the viscoelastic properties measured through the oscillatory rheological tests (Pearson's coefficient for the complex modulus $G^{*}$ taken at $1 \mathrm{~Hz}$ was 0.8 ). For the materials of group $\mathrm{B}$ and $\mathrm{C}$, which showed a weak-gel rheology pattern, stringiness was negatively correlated to the $\eta$ values (Pearson's -0.7 ) because the length of the string formed when the probe was withdrawn from the sample decreased with an increasing material concentration, as the three-dimensional network becomes more and more structured. On the contrary, for the materials of group A, the stringiness values were significantly positively correlated to $\eta$ and $G^{*}$ (Pearson's coefficient $0.7-$ 0.8 ), in accordance with their viscoelastic behavior.

\section{CONCLUSIONS}

The results of this work proved that objective data about rheology and texture obtained, studying the behavior of the aqueous dispersions of the single raw materials, are useful to predict the sensorial properties they can confer to cosmetic products. Our protocol based on rheological and texture analyses, which are significantly correlated, turned out to be a powerful prescreening tool to characterize and categorize each polymeric raw material, according to their applicative specificities. As expected, natural-derived polysaccharides do not reach the mechanical properties of highly crosslinked acrylic polymers such as carbomer, which showed higher physical parameters. Matching the performance of synthetic polymers by replacing them with polysaccharides is difficult because each raw material has its own sensorial specificities and synthetic polymers are artificially created to have a specific performance in formulation. However, some of the acrylic polymers analyzed in this study, which formed aqueous systems with medium-low viscosity and high spreadability, showed some significative similarities with natural polymers. The water dispersions of succinoglycans (N2) and sclerotium gums (N3) had values of firmness and consistency similar to that of polyacrylate cross-polymer-11 (S3). In addition, they showed a weak-gel rheological pattern and similar values of moduli, but they differed from the values of adhesiveness and the damping factor $\tan \delta$ because the acrylic polymer had a more elastic behavior. Carrageenan (N7) showed a rheological trend very similar to that showed by the hydrophobically modified polymer S4, and they both formed soft and elastic gels when dispersed in water. However, the natural-derived polymer N7 did not reach the values of adhesiveness calculated for S4. In conclusion, the combined use of texture analyses and rheology proved to be a simple time and money-saving system to avoid the traditional trial and error approach to the formulation of natural cosmetic products, especially during a revision of the formulation design or the substitution of an ingredient, in reference to the concern around liquid plastics, is required.

\section{EXPERIMENTAL SECTION}

Materials. 13 raw materials recently introduced in the market as rheological modifiers were selected for the study.

Synthetic raw materials are as follows: S1-carbomer (Carbopol Ultrez 10 by Lubrizol), S2-sodium polyacrylate (Cosmedia SP by BASF), S3-polyacrylate cross-polymer-11 (Aristoflex Velvet by Clariant), S4-ammonium acryloyldimethyltaurate/Beheneth-25 methacrylate cross-polymer (Aristoflex Blv by Clariant), and S5-acrylates/C10-30 alkyl acrylate cross-polymer (Pemulen EZ4U by Lubrizol).

The semisynthetic raw material is the SN-hydroxypropyl guar gum (Jaguar HP-105 by Solvay Novecare).

Natural raw materials are the N1-xanthan gum (Satiaxane VPC 911 by Cargill), N2-succinoglycan (Rheozan SH by Solvay Novecare), N3-sclerotium gum (Actigum CS 11 QD by Cargill), N4-C. spinosa gum (Vari Tara by Variati), N5C. siliqua gum (Vari Carruba by Variati), N6-gellan gum (Kelcogel CG-HA by CP Kelco), and N7-carrageenan (Genuvisco CG-131 by CP Kelco). 
Preparation of the Samples. The synthetic polymers $(\mathrm{S} 1-\mathrm{S} 5)$ were dispersed in hot water $\left(70-75^{\circ} \mathrm{C}\right)$ at increasing concentrations by means of the Silverson L5T homogenizer at $2500 \mathrm{rpm}$ until homogeneity. Polymers S1 and S5 needed neutralization with $\mathrm{NaOH}$ solution $10 \% \mathrm{w} / \mathrm{w}$.

Semisynthetic (SN) and natural polymers (N1-N7) were dispersed in water at increasing amounts under continuous stirring with a magnetic stirrer. SN, N1, N2, and N3 were dispersed in cold water. N4, N5, N6, and N7 needed higher temperature to be dispersed $\left(70^{\circ} \mathrm{C}\right.$ for $\mathrm{N} 4, \mathrm{~N} 5$ and $\mathrm{N} 6,45^{\circ} \mathrm{C}$ for N7). Salt was needed for the dispersion of polymers N6 and N7.

The $\mathrm{pH}$ of the dispersions obtained was between 5 and 6 . The sample preparation modality, including the concentration ranges used in this work, is summarized in Table S7.

Texture Analysis. A Texture Analyzer TMS-Pro, from the Food Technology Corporation (Sterling, Virginia, USA), was used equipped with a load cell of $10 \mathrm{~N}$ and a nylon, spherical probe $(2 \mathrm{~cm}$ diameter $)$. The samples were poured in jars with a volume of $50 \mathrm{~mL}$ and a diameter of $5.3 \mathrm{~cm}$. An immersion/ deimmersion test was conducted in triplicates at room temperature: through a vertical displacement, the probe penetrated the samples for $10 \mathrm{~mm}$ at a rate of $1.3 \mathrm{~mm} \mathrm{~s}^{-1}$ and then turned back to its initial position. Software Texture Lab Pro was used to register and display the data. Figure 12

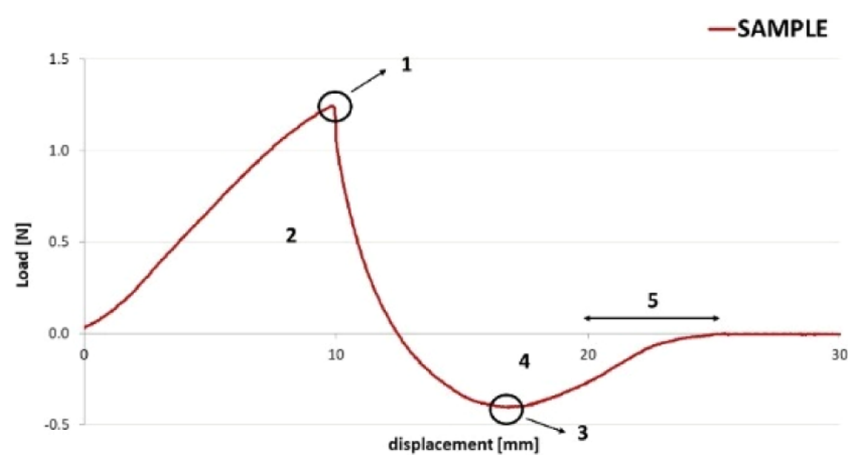

Figure 12. Typical curve obtained with an immersion/deimmersion test, performed with a texture analyzer, and the parameters obtained: firmness (1), consistency (2), cohesiveness (3), adhesiveness, (4) and stringiness (5). Data plotted as load vs cumulative displacement.

shows the texture parameters collected from the curve load (N) vs cumulative displacement ( $\mathrm{mm}$ ): firmness, consistency, cohesiveness, adhesiveness, and stringiness. Firmness is the positive peak, that is, the maximum value of force needed to obtain a deformation and is expressed as newton (N); consistency is the area under the positive curve $(\mathrm{N} \mathrm{mm})$ and represents the total work the sample opposes against the deformation. These two parameters obtained from the first part of the curve are related to the viscosity of the sample and depend on the material molecular weight and crosslinking degree. Cohesiveness is the negative peak $(\mathrm{N})$ registered during the movement of the probe upward and is related to the intramolecular forces of the analyzed material; adhesiveness is the area under the negative portion of the curve $(\mathrm{N} \mathrm{mm})$ and is the work necessary to break the attractive forces established between the surface of the sample and the surface of the instrument probe. These parameters obtained from the negative part of the curve are related to the elastic properties of the sample. Stringiness is the distance $(\mathrm{mm})$ to which the product stretches during the withdrawal of the probe that returns to its initial position. ${ }^{18}$ This parameter describes the viscoelasticity of the product.

Rheological Analyses. The instrument used is the rheometer Physica MCR-101 from Anton Paar. All the analyses were performed both in continuous and oscillatory flow conditions, at a controlled temperature $\left(23 \pm 0.05{ }^{\circ} \mathrm{C}\right)$, using the CP50-1 sensor, which consisted in a parallel plate geometry set at a fixed gap of $0.1 \mathrm{~mm}$. The measurements were conducted in triplicates, ensuring the reproducibility of the results. The sample flow curves were obtained in continuous flow conditions, with a CSR test, by recording viscosity values $(\eta)$ at an increasing shear rate, from 0.001 to $1000 \mathrm{~s}^{-1}$. Fitting the flow curves with the Carreau-Yasuda mathematical model that describes the shear thinning behavior of the material (eq 1 ), the viscosity of the samples in the plateau region (zeroshear viscosity) was calculated, as an indication of the viscosity at rest.

$$
\tau=\eta_{\infty} \dot{\gamma}+\frac{\left(\eta_{0}-\eta_{\infty}\right) \dot{\gamma}}{\left[1+(\lambda \dot{\gamma})^{2}\right]^{1-n / 2}}
$$

The tests performed in oscillatory conditions allowed highlighting the viscoelastic properties of the polymer dispersions. An amplitude sweep test was conducted increasing the strain $(\gamma)$ from 0.01 to $1000 \%$, maintaining the frequency at $1 \mathrm{~Hz}$, in order to identify the LVR. Frequency sweep tests, were performed decreasing the frequency from 10 to $0.01 \mathrm{~Hz}$, at a fixed strain chosen from the LVR. These tests were used to study the inner structure of the dispersion and the trend of the storage $\left(G^{\prime}\right)$ and loss $\left(G^{\prime \prime}\right)$ moduli.

Statistical Analysis. XLSTAT software (Addinsoft, Paris, France) was used to elaborate the data obtained from the analytical tests. PCA was performed on the correlation matrix made with the average values of the textural parameters measured for each sample. This analysis allowed evaluating the differences and the similarities between the raw materials subjected to the analyses. Pearson's correlation coefficient was calculated considering the numerical values of the textural and rheological parameters, to highlight which ones were significantly correlated $(p$-value $<0.05)$.

\section{ASSOCIATED CONTENT}

\section{Supporting Information}

The Supporting Information is available free of charge at https://pubs.acs.org/doi/10.1021/acsomega.0c01306.

List of the rheological modifier used in this work and the dispersion modality for the preparation of samples; numerical values of the textural parameters measured for the samples prepared at increasing concentrations of the raw material of group A; zero shear viscosity $\left(\eta_{0}\right)$ values, calculated with the Carreau-Yasuda model, for the samples prepared with increasing concentrations of the raw materials of group A; numerical values of the textural parameters measured for the samples prepared at increasing concentrations of the raw material of group B; flow curves of the samples prepared with the polymers of group B at $1 \% \mathrm{w} / \mathrm{w}$; zero shear viscosity $\left(\eta_{0}\right)$ values, calculated with the Carreau-Yasuda model, for the samples prepared with increasing concentrations of the raw materials of group B; numerical values of the textural parameters measured for the samples prepared at increasing concentration of the raw material of group C; flow curves of the samples prepared with the 
polymers of group $\mathrm{C}$ at $0.5 \% \mathrm{w} / \mathrm{w}$; and zero shear viscosity $\left(\eta_{0}\right)$ values, calculated with the CarreauYasuda model, for the samples prepared with increasing concentrations of the raw materials of group $\mathrm{C}$ (PDF)

\section{AUTHOR INFORMATION}

\section{Corresponding Author}

Giovanni Tafuro - Department of Pharmaceutical and Pharmacological Sciences, University of Padua, 35131 Padua, Italy; 이이.org/0000-0003-2717-2702;

Email: giovanni.tafuro@unipd.it

\section{Authors}

Alessia Costantini - Unired S.r.l., Spin-off University of Padua, 35131 Padua, Italy

Gianni Baratto - Unifarco S.p.A., 32035 Santa Giustina, Belluno, Italy

Stefano Francescato - Unifarco S.p.A., 32035 Santa Giustina, Belluno, Italy

Alessandra Semenzato - Department of Pharmaceutical and Pharmacological Sciences, University of Padua, 35131 Padua, Italy

Complete contact information is available at:

https://pubs.acs.org/10.1021/acsomega.0c01306

\section{Notes}

The authors declare no competing financial interest.

\section{ACKNOWLEDGMENTS}

The authors would like to acknowledge Dr Giulia Galizia from Unired s.r.l. for the writing assistance and language editing. We also thank the reviewers for their comments and suggestions.

\section{REFERENCES}

(1) Medina-Torres, L.; Calderas, F.; Sanchez-Olivares, G.; NuñezRamirez, D.-M. Rheology of Sodium Polyacrylate as an emulsifier employed in cosmetic emulsions. Ind. Eng. Chem. Res. 2014, 53, 18346-18351.

(2) Bom, S.; Ribeiro, H. M.; Marto, J. Sustainability Calculator: A Tool to Assess Sustainability in Cosmetic Products. Sustainability 2020, 12, 1437.

(3) Bom, S.; Jorge, J.; Ribeiro, H. M.; Marto, J. A step forward on sustainability in the cosmetics industry: A review. J. Clean. Prod. 2019, $225,270-290$.

(4) Schneiders, B.; Anklin, F. The social impact of a cosmetics company. In Sustainability: How the Cosmetics Industry is Greening Up; Sahota, A., Ed.; John Wiley \& Sons, Ltd: London, 2014; pp 47-68.

(5) Grabenhofer, R. L. How Bio-derived Became Sustainable; Cosmetics \& Toiletries, 2019; pp 2-5.

(6) Kentin, E.; Kaarto, H. An EU ban on microplastics in cosmetic products and the right to regulate. RECIEL 2018, 27, 254-266.

(7) Reichmuth, N.; Pedan, V.; Ott, R.; Huber, P. Sensory-driven substitution of acrylate polymers with natural alternatives. IFSCC 2019 Conference Proceedings, 2019.

(8) Gilbert, L.; Picard, C.; Savary, G.; Grisel, M. Impact of Polymers on Texture Properties of Cosmetic Emulsions: A Methodological Approach. J. Sens. Stud. 2012, 27, 392-402.

(9) Estanqueiro, M.; Amaral, M. H.; Sousa Lobo, J. M. Comparison between sensory and instrumental characterization of topical formulations: impact of thickening agent. Int. J. Cosmet. Sci. 2016, 38, 389-398.

(10) Wortel, V. A. L.; Wiechers, J. W. Skin sensory performance of individual personal care ingredients and marketed personal care products. Food Qual. Pref. 2000, 11, 121-127.
(11) Goddard, E. D.; Gruber, J. V. Principles of Polymer Science and Technology in Cosmetics and Personal Care; Marcel Dekker Inc.: New York, 1999; pp 339-403.

(12) Semenzato, A.; Costantini, A.; Baratto, G. Green Polymers in Personal Care Products: Rheological Properties of Tamarind Seed Polysaccharide. Cosmetics 2014, 2, 1-10.

(13) Tadros, T. Application of rheology for assessment and prediction of the long-term physical stability of emulsions. Adv. Colloid Interface Sci. 2004, 108-109, 227-258.

(14) Brummer, R.; Godersky, S. Rheological studies to objectify sensations occurring when cosmetic emulsions are applied to the skin. Colloids Surf. A Physicochem. Eng. Asp. 1999, 152, 89-94.

(15) Nishinari, K.; Kohyama, K.; Kumagai, H.; Funami, T.; Bourne, M. C. Parameters of Texture Profile Analysis. Food Sci. Technol. Res. 2013, 19, 519-521.

(16) Peng, F.; He, S.; Yi, H.; Li, Q.; Xu, W.; Wang, R.; Ma, Y. Physical, textural, and rheological properties of whipped cream affected by milk fat globule membrane protein. Int. J. Food Prop. 2018, 21, 1190-1202.

(17) Liu, H.; Xu, X. M.; Guo, S. D. Rheological, texture and sensory properties of low-fat mayonnaise with different fat mimetics. $L W T-$ Food Sci. Tech. 2007, 40, 946-954.

(18) Tai, A.; Bianchini, R.; Jachowicz, J. Texture analysis of cosmetic/pharmaceutical raw materials and formulations. Int. J. Cosmet. Sci. 2014, 36, 291-304.

(19) Gilbert, L.; Picard, C.; Savary, G.; Grisel, M. Rheological and textural characterization of cosmetic emulsions containing natural and synthetic polymers: relationships between both data. Colloids Surf. A Physiochem. Eng. Asp. 2013, 421, 150-163.

(20) Tamburic, S.; Sisson, H.; Cunningham, N.; Stevic, M. C. Rheological and Texture Analysis Methods for Quantifying Yield Value and Level of Thixotropy. SOFW J. 2017, 143, 24-30.

(21) Tafuro, G.; Costantini, A.; Baratto, G.; Busata, L.; Semenzato, A. Rheological and Textural Characterization of Acrylic Polymer Water Dispersions for Cosmetic Use. Ind. Eng. Chem. Res. 2019, 58, 23549-23558.

(22) Tamburic, S.; Stevic, M.; Sisson, H. Rheological and Texture Analysis Methods for the Assessment of Viscoelasticity. SOFW J. 2018, 144, 20-25.

(23) Prajapati, V. D.; Jani, G. K.; Moradiya, N. G.; Randeria, N. P.; Nagar, B. J.; Naikwadi, N. N.; Variya, B. C. Galactomannan: A versatile biodegradable seed polysaccharide. Int. J. Biol. Macromol. 2013, 60, 83-92.

(24) Rinaudo, M.; Tinland, B. Relation between Molecular Structure and Physicochemical Properties for Some Microbial Polysaccharides. In Novel Biodegradable Microbial Polymers; Dawes, E. A., Ed.; Kluwer Academic Publishers: Norwell, MA, 1990; pp 349-370.

(25) Lapasin, R.; Pricl, S. Rheology of Industrial Polysaccarides, Theory and Application; Blackie Academic \& Professional: London, 1995; pp $32-44$.

(26) Grasdalen, H.; Smidsrød, O. Gelation of Gellan Gum. Carbohydr. Polym. 1987, 7, 371-393.

(27) Blakemore, W. R.; Harpell, A. R. Carrageenan. In Food Stabilizers, Thickeners and Gelling Agents; Imeson, A., Ed.; WileyBlackwell: Oxford, 2010; pp 73-94. 